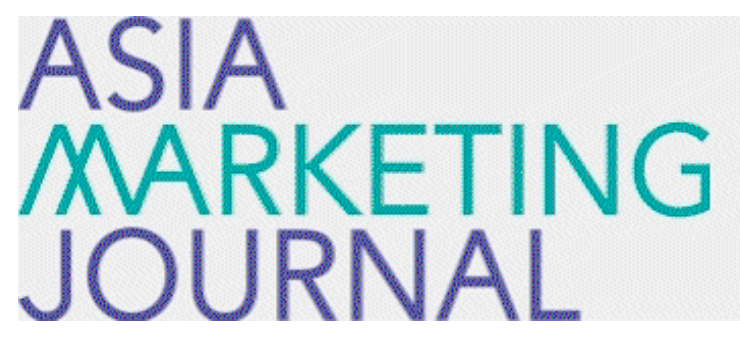

ASIA MARKETING JOURNAL

Volume 15 | Issue 1

Article 8

4-30-2013

\title{
Movie Choice under Joint Decision
}

Young Ju Kim

Jae Hwan Kim

Follow this and additional works at: https://amj.kma.re.kr/journal

Part of the Marketing Commons

\section{Recommended Citation}

Kim, Young Ju and Kim, Jae Hwan (2013) "Movie Choice under Joint Decision," Asia Marketing Journal:

Vol. 15 : Iss. 1 , Article 8.

Available at: https://doi.org/10.53728/2765-6500.1514

This Article is brought to you for free and open access by Asia Marketing Journal. It has been accepted for inclusion in Asia Marketing Journal by an authorized editor of Asia Marketing Journal. 


\title{
Movie Choice under Joint Decision: Reassessment of Online WOM Effect*
}

\author{
Youngju Kim** \\ Jaehwan Kim***
}

This study describes consumers' movie choices in conjunction with other group members and attempts to reassess the effect of the online word of mouth (WOM) source in a joint decision context. The tendency of many people to go to movies in groups has been mentioned in previous literature but there is no modeling research that studies movie choice from the group decision perspective. We found that ignoring the group movie-going perspective can result in a misunderstanding, especially underestimation of genre preference and the impact of the WOM variables. Most of the studies to measure online WOM effects were done at the aggregate level, and the role of online WOM variables(volume vs valence) is mixed in the literature. We postulate that group-level analysis might offer insight to resolve these mixed understanding of WOM effects in the literature.

We implemented the study via a random effect model with group-level heterogeneity. Romance, drama, and action were selected as genre variables: valence and volume were selected as online WOM variables. A choice-based conjoint survey was used for data collection and the models was estimated via Bayesian MCMC method. The empirical results show that (i) both genre and online WOM are important variables when consumers choose movies, especially as group, and (ii) the WOM valence effect are amplified more than the volume effect does as individuals are engaged in group decision.

This research contributes to the literature in several ways. First, we investigate movie choice from a group movie-going perspective that is more realistic and consistent with the market behavior. Secondly, the study sheds new light on the WOM effect. At group-level, both valence and volume significantly affect movie choices, which adds to the understanding of the role of online WOM in consumers movie choice.

Key words: Movie choice, Group Decision, Online Word of Mouth, Entertainment Marketing

\footnotetext{
* We are grateful to Jeff Dotson and participants at KUBS Modeling Lunch seminar for valuable comments.

** Ph.D. Candidate in Marketing, Korea University Business School, Korea(aa0124@korea,ac.kr)

*** Associate Professor of Marketing. Korea University Business School, Korea(jbayes@korea.ac.kr), Corresponding Author
} 


\section{Introduction}

This study attempts to empirically investigate movie choice based on the viewpoint that consumers make their choice as a group rather than individuals. Among the many variables that might influence movie viewing, we specifically focus on the online word-of-mouth effect. Therefore, motivation for the study is twofold - one is understanding movie choice as group decision and the other is reassessing the WOM effect in the motion picture industry.

The tendency of many people to go to movies in groups has been mentioned in previous literature: movie watching is a group activity, shared with friends or family (De silva 1998: Hartmann 2010: Weinberg 2005), and the companion has direct influence on consumer movie choice (Eliashberg et al. 2006). However, there is no modeling research that studies movie choice from the group decision perspective. For marketing researchers, capturing the movie decision through the joint decision framework means more than merely a change in the unit of analysis. This is because there are complicated factors unique to group decision situations. These factors include the shift in the tendency to avoid risk, emergence of polarization (Rao and Steckel 1991), and the relative power of members within the group. Therefore, ignoring the group moviegoing perspective possibly results in misunderstanding the impact of the marketing ac- tivities of movie makers. To correct this misunderstanding requires data collection and statistical inference at the proper unit-of-analysis level, i.e., the group level. We find in our analysis that the effects of movie attributes and preferences are systematically underestimated when ignoring the joint decision perspective in movie choice.

Another issue that we would like to address is the online WOM effects. There are two reasons why online WOM is relevant to this study. First, despite many researchers having investigated online WOM effects at the aggregate level, no studies have been done at the microlevel (Duan et al. 2008a). The WOM effects at group level analysis have not been known to us. Secondly, we have not had a consistent understanding regarding the roles of two representative online WOM variables in the literature - volume and valence. Previous findings are mixed - some say only volume matters, but valence does not (e.g., Duan et al. 2008a: Liu 2006), whereas others report the opposite (e.g., Chintagunta et al. 2010). Both findings are suspicious as the utility of a movie should not significantly increase when many people report negative comments, nor when positive information is only from very few people. We attempt to offer alternative reasons for these conflicts in the literature by reassessing the online WOM effect at the group level. In our study, empirical analysis of online WOM effects shows the following: first, when people 
decide as a group, both valence and volume effects are amplified: second, valence effects are amplified more than are volume effects.

In summary, we propose a modeling study of heterogeneous movie choice under joint decision situations that addresses the challenges listed above. We implemented the study via a Bayesian random effect model. Note that analysis with heterogeneity under the assumption that a person goes to see a movie alone would not be realistic since the data in the real market are more consistent with group decision outcomes. The immediate questions being addressed via this study are whether there is a systematic under (over) estimation of the movie characteristic variables when the investigation is done at the perspective of the group rather than the individual. Our study also revises our understanding of movie consumption, especially regarding the significance of online WOM valence and online WOM volume. Given the most studies in the literature were done to measure those effects on the box-office revenues at the macro level, our study is expected to complement the literature. Also, such reassessment would bring more realistic understanding on the role of online WOM variables, partially solving the mixed findings in the literature.

In the next section, we briefly summarize the literature review on WOM effect in movie studies. In section 3, we describe the model. In section 4 , we explain the data used. In section 5 , the estimated results are presented. We close with concluding remarks and suggestions for future research.

\section{Literature Review}

\section{Word of Mouth in Movie Consumption}

In the motion picture industry, many researchers have focused on the effects of online WOM (Duan et al. 2008b), especially valence (evaluations given by people who have watched the movie) and volume (the total number of posted reviews by people who have watched the movie). However, previous studies dealing with WOM effects in the motion picture industry, mostly conducted at the macro level, have shown mixed results for online WOM effects, according to the model and the basic assumptions used. Liu (2006) used an OLS method to find a temporal relationship between online WOM and box office revenue. The results showed that volume, and not valence, was an important variable for motion picture success. Duan et al. (2008a) used a simultaneous equation system to capture the interdependent relationship between online WOM and box office revenue, and the results also showed that only volume was a significant variable for movie sales. However, when they considered the interaction between volume and valence, the results showed that volume significantly influences movie sales, and 
valence has an indirect influence on movie sales by mediating volume (Duan et al. 2008b). However, Chintagunta et al. (2010) measured the impact of online user reviews, and the results showed that only valence, not volume, matters for movie sales. On the other hand, Dellarocas et al. (2007) used the Bass diffusion model and found that both valence and volume were significant predictors of future movie revenue. When Bae et al, (2010) used Korean movie ratings data for a simultaneous model, they found that volume is not the cause of the movie revenue but the result of the movie revenue.

In the current study, we focus on online WOM effects at the micro-level, i.e., individual and group levels. We anticipate that both valence and volume could be significant. Valence can be translated into an assessment of movie quality by people who saw the movies. High online WOM valence indicates a movie of high quality, and by using this information, customers can reduce the risk of choosing a movie of poor quality. Volume, an indication that many people have already discussed the movie, could suggest popularity, which represents a social cue and tends to reduce the customers' perceived risk (Desarbo et al, 2002) of choosing a movie of poor quality. Also, we expect that parameter estimates of online WOM will be amplified when movie choice is made by a group as opposed to by individuals because people tend to be more sensitive to generally accept- able quality cues when they are engaged in making decision with others.

\subsection{Group Decision}

In the marketing modeling area, group choice researches are divided into two parts: linear model and normative model (Steckel et al. 1991). A linear model is divided into two according to the objectives of the model: to reveal the group choice mechanism or to build the model predicting group choice. For the models with group choice mechanism, Allenby and Arora (1999) conducted research to explain the relative decision power in the group choice situation at the attribute level. Aribarg et al. (2002) built a model to reveal the group decision flow. For the models predicting group choice, Arora (2006) built a sub-sampling approach model to predict group choice with full individual data and partial group data. Aribarg et al. (2010) built a model that can predict the group choice only with individual data. Otherwise, linear models could be divided with the basic assumption of model building: altruistic utility function, maximize utility function, minimize regret function, and maximum aggregation function. On the other stream, there is a normative model. Most research took this perspective based on the theory of cooperative games. One of the most recent examples is Hartmann (2010), who took a game theory approach for estimation and analysis of demand in the context of group 
choice. (If you are interested in more specific group choice research, see two review papers on this area [Adamowicz et al. 2005: Steckel et al 1991] or Zhang et al. [2009]).

\section{Model}

\subsection{Model specification}

Following standard random utility model framework, we assume that each group's choice is driven by the latent utility of the movie. Let subscript $\mathrm{g}, \mathrm{j}, \mathrm{k}$ refer to each group g, movie j and movie attribute $\mathrm{k}$, respectively. The utility of a movie is captured as,

$$
\mathrm{U}_{\mathrm{g}, \mathrm{j}}=\sum_{\mathrm{k}=1}^{\mathrm{K}} \beta_{\mathrm{g}, \mathrm{k}} \mathrm{X}_{\mathrm{j}, \mathrm{k}}+\varepsilon_{\mathrm{g}, \mathrm{j}}
$$

where

$\mathrm{U}_{\mathrm{gj}}$ : utility of group $\mathrm{g}$ for movie alternative $\mathrm{j}$

$\beta_{g, k}$ : sensitivity of group $g$ to movie attribute $\mathrm{k}$

$\mathrm{X}_{j, k}: \mathrm{k}^{\text {th }}$ attribute for movie alternative $\mathrm{j}$

$\varepsilon_{\mathrm{g.j}}:$ random terms

We postulate that the utility of a movie to a group results from the weighting of individual utilities between members in the group. More specifically, the group sensitivity to movie attribute $\mathrm{k}$ would be determined by weighting each individual group member's sensitivity. Following the approach in the literature on group decision making (Aribarg et al, 2002: Arora and Allenby 1999), we model the weighting process focusing on dyads, particularly female-male groups, one of the groups visiting the movie theater most frequently. Thus, in eq. (2), $m$ refers to the male member and $w$ refers to the female member of the group g. ${ }^{1}$

$$
\beta_{\mathrm{g}, \mathrm{k}}=\phi_{\mathrm{g}, \mathrm{k}} \cdot \beta_{\mathrm{g}_{\mathrm{w}}, \mathrm{k}}+\left(1-\phi_{\mathrm{g}, \mathrm{k}}\right) \cdot \beta_{\mathrm{g}_{\mathrm{m}}, \mathrm{k}}
$$

$$
\begin{aligned}
& \text { where } \\
& \beta_{g_{v}, k}: \text { sensitivity of female member of group } g \text { to } \\
& \text { attribute } \mathrm{k} \\
& \beta_{g_{m}, k}: \begin{array}{l}
\text { sensitivity of male member of group } \mathrm{g} \text { to } \\
\text { attribute } \mathrm{k}
\end{array} \\
& \phi_{\mathrm{g}, \mathrm{k}}: \text { : female member influence within group } \mathrm{g} \\
& \quad \text { on sensitivity to attribute } \mathrm{k}
\end{aligned}
$$

In eq. (2), $\phi_{\text {g,k }}$ is the group-specific parameter. To flexibly capture the resulting group $\beta$ as in Arora and Allenby (1999), we did not restrict $\phi$ to be $[0,1]$. In our analysis, therefore, the amplification of an attribute impact is possible when movie choice is made by a group.

\subsection{Likelihood}

We assume random terms in (1) as $\varepsilon_{\mathrm{gj}} \sim \mathrm{EV}$ $(0,1)$. So, the probability that movie $\mathrm{j}$ is se-

1) To get each group members' sensitivity for attribute, we determined the utility of a movie product for each individual first. Utility of a movie for an individual is determined similarly to the group-decision model. 
lected by group $\mathrm{g}$ at time $\mathrm{t}$ is given by the logit form in (3):

$$
P_{g, j}=\frac{\exp \left(\sum_{k=1}^{K} \beta_{g, k} X_{j, k, t}^{\prime}\right)}{\sum_{k=1}^{J} \exp \left(\sum_{k=1}^{K} \beta_{g, k} X_{j, k, t}^{\prime}\right)}
$$

Assuming that there are $\mathrm{G}$ total groups each with $\mathrm{T}_{\mathrm{g}}$ observations, the complete likelihoods for the group choice perspective model ( $\mathrm{L}_{\text {group }}$ ) are as follows:

$$
L_{\text {group }}=\prod_{g=1}^{G}\left(\prod_{t=1}^{T_{g}}\left(\prod_{j=1}^{J}\left(P_{g j, t}\right)^{y_{g j, t}}\right)\right)
$$

where $\mathrm{y}_{\mathrm{g} j \mathrm{t}}=1$ if movie $\mathrm{j}$ was selected at $\mathrm{t}$ and 0 otherwise.

\subsection{Heterogeneity}

In eq. (1) and (2), the parameters are all group-specific. We capture this heterogeneity across the groups through continuous representation of the random effect framework (Allenby and Rossi 1999) as in eq. (5).

$$
\phi_{\mathrm{g}}=\Gamma \mathrm{z}_{\mathrm{g}}+\xi_{\mathrm{g}} \quad \text { where } \xi_{\mathrm{g}} \sim \mathrm{N}\left(0, \Sigma_{\phi}\right)
$$

$Z_{g}$ : group characteristics such as marital status and interest gaps between members

$\Gamma$ : matrix of coefficients that relate the groupparameters to the value of $Z_{g}$

$\Sigma_{g}$ : unobserved heterogeneity among groups

\section{Empirical Analysis}

\subsection{Variables}

We used genre and online WOM as explanatory variables(i.e., $\mathrm{X}$ variables). The three genres of dummy specification are romance, action/thriller, and drama. In this study, valence is defined as the cumulative average rating, which is the most popular format of displaying aggregate feedback information on websites (Duan et al. 2008b), and volume is defined as the log transformation of total number of reviews on the site.

We considered marital status and intra-group film interest gaps (Burns and Granbois 1977) as group characteristic variables(i.e., $z$ variables). Interest was measured by the total number of online movie sites joined by each individual. We calculated the interest gaps by subtracting male value from female value.

\subsection{Data}

Empirical data was collected through a choiceresponse survey for the couples. Potential respondents were invited in pairs to perform two different sessions - one for individual decision and the other for group decision - to choose one among four movies (We used six actual movies each week as stimuli, which were on screen at the time of data collection. In choice 
set given at each task, there are four movies among six movies). For the first session, each member of a group separately selected one movie for themselves, assuming that they would watch the movie alone. They were given seven to eight tasks. Each member was then asked to jointly decide on a movie to watch together. The second session consisted of fifteen tasks. After the two sessions were completed, information on the respondents demographic variables and group characteristics was collected.

The average value of the valence and $\log$ volume for movies in our data were 7.27 (standard deviation (SD): 1.47), and 5.01 (SD: 1.49), respectively.

Respondents were restricted to female-male couples only. A total of 120 couples participated in data collection, 84 unmarried and 36 married. For all 240 participants, the average number of movie related sites joined was 1.60. The average gap of movie related sites joined between group members was 0.1 , varying from -5 to 4

\section{Results}

The posterior distribution of the parameters of our model was estimated via Markov Chain Monte Carlo (MCMC) in the hierarchical Bayesian framework (Rossi, Allenby, and McCullogh 2005).

\subsection{Estimation results}

\subsubsection{Analysis for group-choice level}

In the group choice perspective model, we postulated that the estimate of movie preference $\left(\beta_{g}\right)$ is a function of each individual's original preference $\left(\beta_{g, w}, \beta_{g, m}\right)^{2)}$ and the relative influence between the individuals $\left(\phi_{g}\right)$. The mixing coefficient $\phi$ in eq. (2) reflects the pattern of the influence within the group. As $\phi$ approaches $1(0)$, the female (male) member demonstrates higher influence than the male (female). The estimation results are in table 1. The left-hand side presents the posterior distribution for the mixing coefficients $(\phi)$ and their relation to group characteristics $(\Gamma)$.

On average, females have a higher influence on the group decision process $\left(\Gamma_{11}=0.70, \Gamma_{21}\right.$ $=0.66, \Gamma_{31}=0.60$, and $\left.\Gamma_{41}=0.59\right)$. No variables in group characteristics in the current data were found to explain relative group decision power in this study (see $\Gamma$ in Table 1). Although identifying which variables are (or are not) responsible for group decision dynamics is not the goal of our study, this area might be an interesting avenue for future research. From the covariancelcorrelation matrix in table 1 , we found that groups differ in the magni-

2) To get the groups' sensitivity for each attribute, we need individual group members' sensitivity for each attribute. These estimates are in table 2 . 
$\langle$ Table 1〉 Estimates for Group parameter

\begin{tabular}{c|ccc|cccc}
\hline \multirow{2}{*}{$Z_{\mathrm{g}}$} & \multicolumn{3}{|c|}{ Mean $(\Gamma)$} & \multicolumn{4}{c}{ CovariancelCorrelation $\left(\Sigma_{\phi}\right)$} \\
\cline { 2 - 7 }$\phi$ & $\begin{array}{c}\text { Intercept } \\
\text { Marital Status } \\
\text { (Married=1) }\end{array}$ & $\begin{array}{c}\text { Membership Gap } \\
\text { (Interest Gap) }\end{array}$ & $\begin{array}{c}\text { Genre } \\
\text { Dummy 1 } \\
\text { (Drama) }\end{array}$ & $\begin{array}{c}\text { Genre } \\
\text { Dummy 2 } \\
\text { (Action) }\end{array}$ & $\begin{array}{c}\text { WOM } \\
\text { Valence }\end{array}$ & $\begin{array}{c}\text { WOM } \\
\text { Volume }\end{array}$ \\
\hline $\begin{array}{c}\text { Genre Dummy 1 } \\
\text { (Drama) }\end{array}$ & 0.70 & -0.19 & -0.02 & 0.74 & 0.26 & 0.14 & 0.12 \\
Genre Dummy 2 & $(0.11)$ & $(0.23)$ & $(0.06)$ & $(0.18)$ & & & \\
(Action) & 0.66 & -0.09 & 0.00 & 0.16 & 0.50 & 0.13 & 0.26 \\
WOM & $(0.08)$ & $(0.18)$ & $(0.05)$ & $(0.09)$ & $(0.11)$ & & \\
Valence & 0.60 & -0.06 & 0.01 & 0.11 & 0.08 & 0.87 & 0.04 \\
WOM & $(0.12)$ & $(0.26)$ & $(0.07)$ & $(0.12)$ & $(0.09)$ & $(0.25)$ & \\
Volume & 0.59 & -0.04 & -0.02 & 0.07 & 0.12 & 0.02 & 0.47 \\
\hline
\end{tabular}

tude of the female influence on movie attributes.

After considering group decision power and each group member's sensitivity, we reproduce the group's sensitivity for movie attributes (see left in Table 2). The results show that both genre and online WOM variables used in this study were significant. On average, romance was preferred to both drama and action for the groups $\left(\beta_{1}=-2.62\right.$ and $\left.\beta_{2}=-3.17\right)$, and the effects of WOM valence and WOM volume were both significant in their positive influence on movie choice $\left(\beta_{3}=1.18\right.$ and $\left.\beta_{4}=1.47\right)$. We also found that average groups are persuaded by volume more than by valence.

\subsubsection{Comparison between group choice and individual choice}

The importance of modeling movie choice from different perspectives lies in the possibility that our understanding of which attributes and how much they influence movie choice can be biased, depending on the approach we employ. The first two columns in table 2 show $\beta$ 's from a group choice and an individual choice perspective, respectively. The third and fourth columns represent individual parameters for female and male groups, respectively. There are interesting findings.

First, sensitivities for all movie-related attributes from a group movie-going perspective are systematically higher than those from an individual perspective. This means that in group situations, people are generally more sensitive to the genre and online WOM than in individual choice situations. Therefore, if movie marketers rely only on an individual decision model, they will underestimate the importance 
〈Table 2〉 Comparison on $\beta$ estimates: Group vs. Individual Posterior Mean (Standard deviation)

\begin{tabular}{c|c|c|cc}
\hline \multirow{2}{*}{$\beta$} & \multirow{2}{*}{ Group } & \multicolumn{3}{|c}{ Individual } \\
\cline { 3 - 5 } & & $\begin{array}{c}\text { Individual } \\
\text { (overall) }\end{array}$ & $\begin{array}{c}\text { Female } \\
(\mathrm{W})\end{array}$ & $\begin{array}{c}\text { Male } \\
(\mathrm{m})\end{array}$ \\
\hline $\begin{array}{c}\text { Genre Dummy 1 } \\
\text { (Drama) }\end{array}$ & $-2.62^{*}$ & $-1.33^{*}$ & $-2.11^{*}$ & -0.55 \\
& $(0.46)$ & $(0.28)$ & $(0.31)$ & $(0.44)$ \\
Genre Dummy 2 & $-3.17^{*}$ & $-2.25^{*}$ & $-5.03^{*}$ & 0.53 \\
(Action) & $(0.48)$ & $(0.37)$ & $(0.53)$ & $(0.48)$ \\
WOM & $1.18^{*}$ & $0.89^{*}$ & $0.82^{*}$ & $0.97^{*}$ \\
Valence & $(0.14)$ & $(0.08)$ & $(0.09)$ & $(0.11)$ \\
WOM & $1.47^{*}$ & $1.35^{*}$ & $0.97^{*}$ & $1.73^{*}$ \\
Volume & $(0.16)$ & $(0.13)$ & $(0.14)$ & $(0.20)$ \\
\hline
\end{tabular}

* $95 \%$ confidence region does not include zero.

of movie attributes (genre) and the effects of online WOM.

Second, a group polarization effect (e.g.. Rao and Steckel 1991) is observed. Two group sensitivity parameters, valence and drama dummy, exceed the boundaries between female and male. Specifically, people become more sensitive to others' ratings of the movies (i.e., valence) on average since the group level WOM valence effect is greater than the effect for both female and male $(1.18>\max [0.82,0.97])$. Similar results hold for preference changes for the drama dummy. In this case, the group sensitivity is below the lower boundary between female and male $(-2.62<\min [-2.11$, $-0.55]$ ). The presence of this group polarization phenomenon implies again that a joint decision framework is relevant for movie studies.

\subsection{On the effect of WOM sources on movie choice}

In this section, we focus on the results of online WOM effects, one of the main goals of the study. Empirical analysis of online WOM effects shows the following: first, when people decide as a group, both valence and volume effects are amplified: second, valence effects are amplified more than are volume effects. We further elaborate these findings below.

\subsubsection{WOM effects under Joint decision}

When selecting a movie, why do people become more sensitive to both valence and volume in groups than as individuals? As noted in table 2, there is a systematic shift on average 
in both WOM impacts $((1.18,1.47)>(.89$, 1.35)). Group risk aversion might be one possible explanation for the shift. People tend to avoid risk more in group decision situations (Zajonc et al. 1968). Therefore, it is possible that people try to avoid choosing a poor quality movie as a group more than as individuals. Both online WOM valence and WOM volume decrease movie choice risk because they allow decision makers to justify movie quality.

Also, people would perceive higher risk in group choice than in individual choice. The perceived risk is determined by the importance of the choice taken and the uncertainty of the outcome of group satisfaction (Cunningham 1967: Taylor 1974). In the group choice situation, the uncertainty level of the outcome increases because people cannot predict the partner's taste. Thus, the higher motivation to reduce risk leads them to rely more on external information (Beatty and Smith 1987).

Another question is why the importance of valence is greatly amplified relative to volume. Moving from individual choice perspective to group choice perspective, the impact of valence is increased by $33 \%(1.33=1.18 / 0.89)$ whereas volume is increased by only $9 \% \quad(1.09=1.47 /$ 1.35). Such differences may be attributed to the characteristics of each WOM variable. Although both contribute to risk reduction, valence does so by directly signaling movie quality, i.e., viewers' overall evaluation for the movie (e.g., Dellarocas et al, 2007), whereas volume does so by being a social cue (DeSarbo et al. 2002). Because valence has more direct information than volume has, valence might reduce perceived risk more than volume does.

\subsection{Model Comparison and Predictive Performance}

Does the group choice model predict choice better than the individual choice model? Comparing individual choice and group choice models shows

〈Table 3〉 Model Fit Comparison

\begin{tabular}{ll|ccc}
\hline \hline \multirow{2}{*}{ Model } & \multirow{2}{*}{} & \multirow{2}{*}{ In-sample fit $^{\mathrm{a}}{ }^{2}$} & \multicolumn{2}{c}{ Predictive sample fit } \\
\cline { 3 - 5 } Individual choice perspective $^{\mathrm{b}}$ & & - & Hit Probability & Hit Rate \\
\hline \multirow{2}{*}{ Group choice perspective } & Multiplicative $^{\mathrm{c}}$ & 579.43 & 0.5 & 0.52 \\
& Additive & 2625.47 & 0.68 & 0.72 \\
& Mixture & 6784.95 & 0.59 & 0.54 \\
& & &
\end{tabular}

${ }^{a}$ We use Deviance Information Criteria (DIC), followed by Gelman et al. (2004).

b We predicted group choice using individuals' parameter estimates, individuals who are identified in a group.

$$
\frac{\sum_{i=1}^{120} \mathrm{I}\left(\hat{y}_{\mathrm{B}_{i \mathrm{i}}}=\mathrm{y}_{\mathrm{g}_{i}}\right)+\sum_{i=1}^{120} \mathrm{I}\left(\hat{\mathrm{y}}_{\mathrm{g}_{\mathrm{i}_{\mathrm{im}}}}=\mathrm{y}_{\mathrm{g}_{i}}\right)}{240}
$$

${ }^{\mathrm{C}}$ Model used in current study 
that the group choice model outperforms the individual choice model. The predictive performance is in table 3. Both hit probability (.68 $>.50)$ and hit rate $(.72>.52)$ indicate the same conclusion.

The current model assumes that each group determines the utility of the movie following a multiplicative rule, integrating preference at the attribute level. One can suspect that there may be other rules which capture the integration of individual utilities To address whether the group choice rule represented by eq. (2) is useful to predict the group's movie choice better than possible other rules, we employ two alternative rules as benchmarks - an additive rule and a mixture rule - and fit those models against the same data and check the predictive performance. Note that these benchmark rules assume that group members are not engaged in the process of forming an integrated preference for attributes.

Benchmark 1: (Additive)

$$
P_{g, j}=\frac{\phi_{g} \exp \left(X_{j}^{\prime} \beta_{w}\right)+\left(1-\phi_{g}\right) \exp \left(X_{j}^{\prime} \beta_{m}\right)}{\sum_{k=1}^{j} \phi_{g} \exp \left(X_{k}^{\prime} \beta_{w}\right)+\left(1-\phi_{g}\right) \exp \left(X_{k}^{\prime} \beta_{m}\right)}
$$

Benchmark 2: (Mixture)

$$
P_{g, j}=\phi_{g} \frac{\exp \left(X_{j} \beta_{w}\right)}{\sum_{k=1}^{j} \exp \left(X_{k}^{\prime} \beta_{w}\right)}+\left(1-\phi_{g}\right) \frac{\exp \left(X_{j} \beta_{m}\right)}{\sum_{k=1}^{j} \exp \left(X_{k}^{\prime} \beta_{m}\right)}
$$

In comparison with other group-choice rules, the current model outperforms those bench- marks in both in-sample and predictive fit. Hit probability (.68> $\max \{.59$ (additive rule), .58 (mixture rule) $\})$ and hit rate $(.72>\max \{.54$ (additive rule), .63(mixture rule)\}) are again in consensus. Therefore, we see that the multiplicative rule better predicts the group choice in our data.

\section{Concluding Remarks}

This study makes the following contributions to the literature. First, we studied movie consumption based on a group moviegoing perspective, which is consistent with market behavior. We found that genre preference and the effect of WOM in group decision making are different from those in individual decisions: the effect of all variables we used were amplified and some of them revealed a group polarization effect. Thus, if marketers predict movie demand assuming individual movie choice, they would understate the market responses to their marketing actions.

Secondly, our study adds to the understanding of online WOM effects in the literature. Our study showed that both online WOM valence and online WOM volume are significant drivers of movie success, and these effects are stronger when people make a group decision. This is new to the literature. A previous study at the macro level suggested that "business shall 
focus more on the mechanisms that facilitate dispersion of underlying word-of-mouth exchange rather than try to influence online ratings" (Duan et al. 2008a p.1015). In contrast, we make the new policy suggestion that movie marketers should focus on both online WOM valence and volume.

For future research, we would like to suggest the following opportunities. First, developing a model to capture longitudinal patterns in group decisions is one interesting avenue. In group decision making, decision history plays a significant role (Corfman et al. 1990). The group member who demonstrated higher power in the previous decision period may have less power in the current decision period than other group members. Because moviegoing is often a serial activity with changing choice sets, examining longitudinal choice history of a decision unit could be interesting.

Second, it will be necessary to generalize the settings in group choice. In this study, we restricted our attention to female-male relationships, but the type of relationship can be $\mathrm{ex}^{-}$ tended in the future study.

〈Received March 5. 2013〉

〈Revised April 3. 2013〉

〈Accepted April 8. 2013〉

\section{References}

Adamowicz, Wiktor, Michel Hanemann, Joffre Swait, Reed Johnson, David Layton, Michel Regenwetter, Torsten Reimer, and Robert Sorkin (2005), "Decision Strategy and Structure in Households: A "Groups" Perspective," 16 (3), Marketing Letters, 387-399. Allenby, Greg M. and Peter E. Rossi (1999). "Marketing Models of Consumer Heterogeneity," Journal of Econometrics, 89, 5778.

Aribarg. Anocha, Neeraj Arora and H. Onur Bodur (2002), "Understanding the Role of Preference Revision and Concession in Group Decisions," Journal of Marketing Research. 39 (3), 336-349.

Aribarg, Anocha, Neeraj Arora, and Moon Young Kang (2010), "Predicting Joint Choice using Individual Data," Marketing Science, 29 (1), 139-157.

Arora, Neeraji (2006), "Estimating Joint Preference: A Sub-sampling Approach," International Journal of Research in Marketing. 23, 409-418.

Arora, Neeraj and Greg M. Allenby (1999), "Measuring the Influence of Individual Preference Structures in Group Decision Making," Journal of Marketing Research. 36 (4), 476-87.

Bae, Jungho, Bum Jun Shim and Byung-Do Kim (2010), "Simultaneous Effect between 
eWOM and Revenues: Korea Movie Industry," Asian Marketing Journal, 12 (2), 1-25.

Beatty, Sharon E. and Scott M. Smith (1987), "External Search Effort: an Investigation across Several Product Categories," Journal of Consumer Research, 14 (1), 83-95.

Burns, Alvin C. and Donald Granbois (1977), "Factors Moderating the Resolution of Preference Conflict in Family Automobile Purchasing," Journal of Marketing Research, 14 (February), 77-86.

Chintagunta, Pradeep K., Shyam Gopinath, and Sriram Venkataraman (2010), "The Effects of Online User Reviews on Movie Box Office Performance: Accounting for Sequential Rollout and Aggregation Across Local Markets," Marketing Science, Articles in Advance, 1-14.

Corfman, K. P., D. R Lehmann and J. H. Steckel (1990), "Longitudinal Patterns of Group Decisions: An Exploratory Analysis," Multivariate Behavioral Research, 25, 249273.

Cunningham, S.M. (1967), "The major dimensions of perceived risk," Risk Taking and Information Handling in Consumer Behavior, Harvard University Press, Boston, MA, 82-108.

De silva, Indra (1998), "Consumer Selection of Motion Pictures," The Motion Picture Mega Industry, Needham Heights, MA: Allyn and Bacon Publishing Inc.
Dellarocas, Chrysanthos, Xianoquan Zhang, and Neveen F.Awad (2007), "Exploring the Value of Online Product Reviews in Forecasting Sales: The Case of Motion Pictures," Journal of Interactive Marketing. 21 (4), 23-45.

DeSarbo, Wayne S., Juyoung Kim, S. Chan Choi, and Melinda Spaulding (2002), "A Gravity-based Multidimensional Scaling Model for Deriving Spatial Structures Underlying Consumer Preference/Choice Judgments," Journal of Consumer Research, 29 (1), 91-100.

Duan, Wenjing, Bin Gu, and Andrew B. Whinston (2008a), "Do online reviews matter? - An Empirical Investigation of Panel Data", Decision Support Systems, 45, 1007-1016.

Duan, Wenïng, Bin Gu, and Andrew B. Whinston (2008b), “The Dynamics of Online Wordof-mouth and Product Sales-An Empirical Investigation of the Motion Picture Industry," Journal of Retailing, 84, 233-242.

Eliashberg, Jehoshua, Anita Elberse, and Mark A. A. M. Leenders (2006), "The motion picture industry: Critical issues in practice, current research, and new research directions," Marketing Science,25 (6), 638-661. Gelman, Andrew, John B. Carlin, Hal S. Stern, and Donald B. Rubin (2004), Bayesian data analysis, Boca Raton: Chapman \& Hall.

Hartmann, Wesley (2010), "Demand Estimation with Social Interactions and the Implications for Targeted Marketing," Marketing Science, 
29 (4), 585-601.

Liu, Yong (2006), “WOM for Movies: Its Dynamics and Impact on Box Office Receipts," Journal of Marketing, 70 (3), 74-89.

Rao, Vithala R. and Joel H. Steckel (1991), “A Polarization Model for Describing Group Preferences," Journal of Consumer Research, 18 (June), 108-118.

Rossi, Peter, Greg M. Allenby, and Robert McCulloch (2005), Bayesian Statistics and Marketing, John Wiley \& Sons Ltd.

Steckel, Joel H, Kim P. Corfman, David J. Curry, Sunil Gupta, and James Shanteau (1991), "Prospects and Problems in Modeling Group Decisions," Marketing Letters, 231-240.

Taylor, J.W. (1974), "The Role of Risk in Consumer Behavior," Journal of Marketing. 38 (2), 54-60.
Weinberg. Charles. B (2005), "Profits out of the picture: Research issues and revenue sources beyond The North American box office," C. C. Moul, ed. A Concise Handbook of Movie Industry Economic, Cambridge University Press, New York, 163 203.

Zajonc, Robert B., Rober. J. Wolosin, Myrna A. Wolosin, and And Steven J. Sherman (1968), "Individual and Group Risk-Taking in a Two Choice Situation," Journal of Experimental Social Psychology, 4, 89-106.

Zang, Junyi, Masashi Kuwano, Backin Lee, and Akimasa Fujiwara (2009), "Modeling Household Discrete Choice Behavior Incorporating Heterogeneous Group DecisionMaking Mechanisms," Transportation Research Part B, 43, 230-250. 